\title{
From the Olfactory Bulb to Higher Brain Centers: Genetic Visualization of Secondary Olfactory Pathways in Zebrafish
}

\author{
Nobuhiko Miyasaka, ${ }^{1}$ Kozo Morimoto, ${ }^{1}$ Tatsuya Tsubokawa, ${ }^{3}$ Shin-ichi Higashijima, ${ }^{4}$ Hitoshi Okamoto, ${ }^{2}$ and \\ Yoshihiro Yoshihara ${ }^{1}$ \\ Laboratories for ${ }^{1}$ Neurobiology of Synapse and ${ }^{2}$ Developmental Gene Regulation, RIKEN Brain Science Institute, Wako, Saitama 351-0198, Japan, \\ ${ }^{3}$ Department of Biology, Keio University, Yokohama, Kanagawa 223-8521, Japan, and ${ }^{4}$ National Institute of Natural Sciences, Okazaki Institute for \\ Integrative Bioscience, National Institute for Physiological Sciences, Okazaki, Aichi 444-8787, Japan
}

In the vertebrate olfactory system, odor information is represented as a topographic map in the olfactory bulb (OB). However, it remains unknown how this odor map is transferred from the $\mathrm{OB}$ to higher olfactory centers. Using genetic labeling techniques in zebrafish, we found that the $\mathrm{OB}$ output neurons, mitral cells (MCs), are heterogeneous with respect to transgene expression profiles and spatial distributions. Tracing MC axons at single-cell resolution revealed that (1) individual MCs send axons to multiple target regions in the forebrain; (2) MCs innervating the same glomerulus do not necessarily display the same axon trajectory; (3) MCs innervating distinct glomerular clusters tend to project axons to different, but partly overlapping, target regions; (4) MCs innervating the medial glomerular cluster directly and asymmetrically send axons to the right habenula. We propose that the topographic odor map in the $\mathrm{OB}$ is not maintained intact, but reorganized in higher olfactory centers. Moreover, our finding of asymmetric bulbo-habenular projection renders the olfactory system an attractive model for the studies of brain asymmetry and lateralized behaviors.

\section{Introduction}

Sensory stimuli in the external world are represented as topographic maps in the internal brain. In the vertebrate olfactory system, molecular features of odorants are mapped on the surface of the olfactory bulb $(\mathrm{OB})$ as spatial arrangements of functional neuropil units called glomeruli (Mori et al., 1999). Two basic principles underlie the establishment of odor map in the OB: "one neuron-one receptor rule" (Chess et al., 1994; Malnic et al., 1999; Serizawa et al., 2003) and "axon convergence to specific glomeruli" (Ressler et al., 1994; Vassar et al., 1994; Mombaerts et al., 1996). Individual olfactory sensory neurons (OSNs) select only one odorant receptor (OR) for expression from a large repertoire and OSNs expressing the same OR converge axons onto a few target glomeruli, creating the odor maps (Rubin and Katz, 1999; Uchida et al., 2000).

Odor information transmitted from OSNs to the OB is subsequently conveyed via axons of mitral cells (MCs) to higher olfactory centers, where the odor information is translated to elicit appropriate cognitive, emotional, hormonal, and behavioral responses. One of the major questions in olfactory research is how

\footnotetext{
Received Jan. 9, 2009; revised Feb. 25, 2009; accepted March 9, 2009.

This work was supported in part by a Grant-in-Aid for Scientific Research (C) and a Grant-in-Aid for Scientific Research on Priority Area (Extracellular Environment) to N.M., and a Grant-in-Aid for Scientific Research (B) and a Grant-in-Aid for Scientific Research on Priority Area (Cellular Sensor) to Y.Y. from the Ministry of Education, Culture, Sports, Science, and Technology of Japan. We thank Reinhard W. Köster (California Institute of Technology) for pBGal4VP16 and pBUASEIB plasmids, Stephen J. Smith (Stanford University) for Synaptophysin-GFP cDNA, Min Kyun Park (The University of Tokyo) for anti-GnRH antibody, Marc Ekker (University of Ottawa) for Tg(1.4dlx5a-dlx6a:GFP) transgenic fish, and members of the Yoshihara laboratory for fish care and discussion.

Correspondence should be addressed to Dr. Yoshihiro Yoshihara, Laboratory for Neurobiology of Synapse, RIKEN Brain Science Institute, 2-1 Hirosawa, Wako, Saitama 351-0198, Japan. E-mail: yoshihara@brain.riken.jp. DOI:10.1523/JNEUROSCI.0118-09.2009

Copyright $\odot 2009$ Society for Neuroscience $\quad$ 0270-6474/09/294756-12\$15.00/0
}

the topographic odor map is represented in higher olfactory centers. To answer this question, it is essential to analyze in detail the neuroanatomy of individual MCs with special reference to the dendritic innervation of glomeruli and the axonal trajectory to target regions. Recent studies in Drosophila demonstrated that the projection neurons (equivalent to $\mathrm{MCs}$ ) innervating the same glomerulus in the antennal lobe (equivalent to the $\mathrm{OB}$ ) exhibit stereotypic branching patterns of axons in the mushroom body and lateral horn of the protocerebrum (equivalent to the olfactory cortex), and that the projection neurons innervating different glomeruli display distinct but overlapping distributions of axonal arbors (Marin et al., 2002; Wong et al., 2002; Jefferis et al., 2007; Lin et al., 2007). These findings suggested that the logic of information transfer of the secondary olfactory pathway differs from that of the primary olfactory pathway in Drosophila. In vertebrates, however, axonal trajectories of individual MCs innervating defined glomeruli remain to be elucidated.

Zebrafish has become a fascinating vertebrate model system for olfactory research. Neural activity imaging studies (Friedrich and Korsching, 1997, 1998) demonstrated the existence of odor map in the zebrafish OB. We have recently provided several lines of evidence that the two basic principles (one neuron-one receptor rule; $\mathrm{OSN}$ axon convergence to target glomeruli) underlying the establishment of $\mathrm{OB}$ odor map are essentially preserved also in zebrafish (Sato et al., 2007). Here we identified gene promoters driving transgene expression in MCs. Transient mosaic expression of a fluorescent protein directed by these promoters allowed us to trace axon trajectories of individual MCs at single-cell resolution in larval zebrafish. Our results suggest that the odor map in the $\mathrm{OB}$ is not maintained intact, but reorganized in higher olfactory centers. Moreover, we found that a subpopulation of 
MCs project axons to the telencephalic fields bilaterally and further to the right habenula directly and asymmetrically.

\section{Materials and Methods}

Zebrafish. Zebrafish, Danio rerio, were maintained at $28.5^{\circ} \mathrm{C}$ on a $14 \mathrm{~h}$ light $/ 10 \mathrm{~h}$ dark cycle and embryos were collected by natural spawning. Embryos for imaging experiments were treated with $0.002 \%$ $\mathrm{N}$-phenylthiourea starting at $12-14 \mathrm{~h}$ postfertilization (hpf) to inhibit pigment formation. Two transgenic zebrafish lines, $\operatorname{Tg}\left(\mathrm{OMP}^{2 \mathrm{k}}\right.$ :lynmRFP) ${ }^{\text {rw035a }}$ and $\mathrm{Tg}\left(\right.$ TRPC2 $^{4.5 \mathrm{k}}$ :gap-Venus) ${ }^{\text {rw037a }}$, were described previously (Sato et al., 2005) and are abbreviated as OMP:lyn-RFP and TRPC2:gap-Venus, respectively, in this study.

Generation of transgenic zebrafish. Genomic DNA fragments covering $\sim 10 \mathrm{~kb}$ region upstream of the translation start site of $l h \times 2 a$ gene (GenBank accession number, FJ749249) were obtained by two sets of PCR. The distal $(\sim 8 \mathrm{~kb})$ and proximal $(\sim 4 \mathrm{~kb})$ fragments with an $\sim 2 \mathrm{~kb}$ overlap were separately amplified with primers (PCR1: 5'-TGA ATT GGG AGC CCT AAA CAA TTC CAG CC-3' and 5' -GTG ATA TAG AGA AAA TAA CTC CTT TCT TGC CGC AC-3'; PCR2: 5' -CAA CTG TTT GTG TGG GCC TCA AAA TTA GGG CTG TGC-3' and $5^{\prime}$-GAT TCA CCA ATC CCA GGA CGA GCC GCA AGG-3'). To generate plhx2a: GFP-S, the proximal fragment was first inserted into the SmaI site of pEGFP-S, in which the expression cassette of pEGFP-1 (Clontech) was flanked at both ends by inverted I-SceI sites, and then the overlapping region upstream from the SpeI site was excised and replaced with the distal $\sim 8 \mathrm{~kb}$ fragment. The enhanced green fluorescent protein (EGFP) gene of plhx2a:GFP-S was excised with BamHI and NotI and replaced with genes encoding membrane-tethered EYFP (gap-YFP), Synaptophysin-EGFP fusion protein (Syp-GFP), and GAL4-VP16 fusion protein to generate plhx2a:gap-YFP-S, plhx2a:Syp-GFP-S and plhx2a: GAL4-VP16-S, respectively. Approximately $5 \mathrm{~kb}$ fragment upstream of the translation start site of tbr2a (eomes1/eomesa) gene was amplified from zebrafish genomic DNA by PCR with primers ( $5^{\prime}$-TTA AAA CAA CCC AGC ATT TTT AGA TGG GGA-3' and 5' ${ }^{\prime}$-TCT TCA CTG TGC TGA TAA AGG GAT GAG GAT-3'), and subcloned between the PstI and SacII sites of pEGFP-S to generate ptbr2a:GFP-S. The EGFP-poly(A) cassette of ptbr2a:GFP-S was excised with SacII and AflII and replaced with the DsRed-Express-poly(A) cassette of pDsRed-Express-1 (Clontech) to generate ptbr2a:DsRed-S. To generate stable transgenic lines, circular plasmid DNA at a concentration of $20 \mu \mathrm{g} / \mathrm{ml}$ in $0.5 \times$ I-SceI buffer (New England Biolabs) containing 0.05\% Phenol Red was coinjected with I-SceI meganuclease (0.3 U/ $\mu$ l, New England Biolabs) into one-cell stage embryos. We established two transgenic lines for lhx2a: GFP, two for lhx2a:gap-YFP, one for lhx2a:Syp-GFP, and one for tbr2a: DsRed. For lhx2a:GFP and lhx2a:gap-YFP fish, the line with brighter fluorescence was used in this study, because the expression pattern of fluorescent protein for each construct was essentially the same between the lines. Generation of transgenic lines carrying vglut2a:GFP and vglut2a:loxP-DsRed-loxP-GFP BAC transgenes, in which EGFP and floxed DsRed followed by EGFP were introduced into the vglut $2 a$ (vglut2.1) gene locus, respectively, will be described elsewhere.

Immunohistochemistry. Whole-mount immunohistochemistry was performed essentially as described previously (Miyasaka et al., 2005). Larvae (4-7 dpf) were fixed and permeabilized appropriately for each antibody as follows. For double staining of GFP with tyrosine hydroxylase $(\mathrm{TH})$, gonadotropin-releasing hormone $(\mathrm{GnRH})$, and SV2, larvae were fixed in $10 \%$ formalin (3.7\% formaldehyde, $0.8 \% \mathrm{MeOH})$ in PBS for $30 \mathrm{~min}$ at room temperature (RT) and then in $2 \%$ trichloroacetic acid (TCA) in PBS for $2.5 \mathrm{~h}$ at RT. The fixed larvae were subsequently treated with acetone at $-20^{\circ} \mathrm{C}$ for $7 \mathrm{~min}$ for $\mathrm{TH}$ and $\mathrm{GnRH}$ or with collagenase ( 1 $\mathrm{mg} / \mathrm{ml}$, Sigma) at $37^{\circ} \mathrm{C}$ for $20 \mathrm{~min}$ for SV2. For double staining of membrane-tethered fluorescent proteins with SV2, GTP-binding protein $\alpha$ subunit o (Go), microtubule-associated protein 2 (MAP2), and OCAM, larvae were fixed in 2\% TCA in PBS for $3 \mathrm{~h}$ and subsequently treated with acetone. For double staining of GFP with $\gamma$-aminobutyric acid (GABA), larvae were fixed in $4 \%$ paraformaldehyde (PFA) in PBS for $30 \mathrm{~min}$ and then in $2 \%$ TCA in PBS for $2.5 \mathrm{~h}$. The fixed larvae were subsequently treated with collagenase at $37^{\circ} \mathrm{C}$ for $90 \mathrm{~min}$. Immunohistochemistry of adult brain sections was performed as described previ- ously (Miyasaka et al., 2005) with the following modification of tissue preparation. Adult fish were decapitated and the heads were immersed in $4 \%$ PFA and $15 \%$ saturated picric acid in phosphate buffer at $4^{\circ} \mathrm{C}$ for $2 \mathrm{~h}$. The brains were dissected out and immersed overnight at $4^{\circ} \mathrm{C}$ in the same fixative. The following primary antibodies were used: rat monoclonal anti-GFP antibody (1:1000, Nacalai Tesque); rabbit polyclonal antiDsRed antibody (1:300, Clontech); mouse monoclonal anti-SV2 antibody (1:1000, Developmental Studies Hybridoma Bank); rat polyclonal anti-GABA antibody (1:200, Biomol); mouse monoclonal anti-TH antibody (1:200, Millipore Bioscience Research Reagents); mouse monoclonal anti-GnRH antibody (1:1000, LRH13, a gift from Dr. Min Kyun Park, The University of Tokyo, Tokyo, Japan); rabbit polyclonal anti-Go antibody (1:1000, MBL); mouse monoclonal anti-MAP2 antibody (1:100, Ap-20, BD PharMingen); rabbit polyclonal-neuropeptide Y (NPY) antibody (1:1000, Sigma); rabbit polyclonal anti-OCAM antibody (rabbit IgG, $1.4 \mu \mathrm{g} / \mathrm{ml}$ ). For generating the anti-OCAM antibody, a synthetic C-terminal peptide (20 amino acid residues) of zebrafish OCAM was conjugated with keyhole limpet hemocyanin, and a rabbit was immunized with the conjugate (Sawady Technology). The generated antiserum was subjected to immunoaffinity chromatography with peptide-coupled resin for purification of OCAM-specific antibodies (Sawady Technology).

Image analysis. Live and immunostained larvae were imaged on an inverted microscope (Olympus IX81) equipped with FV500 or FV1000 confocal laser-scanning system through $40 \times$ water-immersion objective (numerical aperture, 0.9; Olympus PlanApo). Three-dimensional projections were generated using Imaris software (Bitplane). Wholemounted adult brains were imaged under a wide-field fluorescence microscope (Olympus MVX10). Composite in-focus images of adult brains were obtained using Dynamic Eye software (Mitani) from $\sim 20$ images at different focal planes.

Genetic single-mitral cell labeling. A mixture of two plasmids, lhx2a(or tbr2a):GAL4-VP16 driver and UAS:gap-YFP responder plasmids (10 $\mu \mathrm{g} / \mathrm{ml}$ each), was injected into one- to four-cell stage embryos. Injected larvae were screened at $3 \mathrm{dpf}$ by confocal microscopy to identify the larvae with single-labeled MCs. The axon trajectories of labeled mitral cells were examined in live larvae by confocal microscopy at $4 \mathrm{dpf}$ and again at $7 \mathrm{dpf}$. After the live imaging, the larvae were fixed and stained with anti-GFP and anti-SV2 antibodies to identify glomeruli innervated by the labeled MCs.

\section{Results}

Generation of transgenic zebrafish with $l h x 2 a$ gene promoter

We previously cloned three members of the zebrafish $l h \times 2 / 9$ gene family (Ando et al., 2005) that encode LIM-homeodomain proteins orthologous to Drosophila Apterous and reported the detailed characterization of the two genes: $l h \times 2 b$ (previously called $l h \times 2)$ and $l h \times 9$. In this study, we focused on the third gene: $\operatorname{lh} \times 2 a$ (supplemental Fig. 1, available at www.jneurosci.org as supplemental material). The expression of $\ln \times 2 a$ mRNA was highly restricted to early born OSNs and their progenitors in the developing olfactory placode/epithelium from 14 to $48 \mathrm{hpf}$ (supplemental Fig. 2, available at www.jneurosci.org as supplemental material). To visualize these neurons, we obtained $\sim 10 \mathrm{~kb}$ genomic DNA fragment upstream of the $\operatorname{lh} \times 2 a$ gene as a transcriptional promoter and generated stable lines of transgenic zebrafish with GFP as a reporter (lhx2a:GFP) (Fig. 1A,B).

GFP expression was first detected in the olfactory placode at $24 \mathrm{hpf}$ (data not shown) and became gradually restricted to a small subpopulation of OSNs, which are localized to the apicocentral portion of the bowl-shaped olfactory organ (Fig. $1 C-C^{\prime \prime}$ ). At $\sim 30$ hpf, GFP expression started in a small number of neurons in the OB (Fig. 1C). As development proceeds, the number of GFP-positive OB neurons increased and remained high in adult fish (Figs. $\left.1 C-C^{\prime \prime}, 7\right)$. As the GFP expression patterns were essentially the same between two independent lines (data not shown), the ectopic transgene expression in the OB neurons would not be 
due to positional effects of transgene integration sites, but due to promoter activities present within the $10 \mathrm{~kb}$ upstream region of the zebrafish $l h x 2 a$ gene. Thus, the $10 \mathrm{~kb}$ upstream sequence of $l h \times 2 a$ gene was insufficient to recapitulate the spatiotemporal expression pattern of endogenous lhx2a. GFP expression was detected not only in a small population of OSNs in the olfactory epithelium (OE), but also in a subset of neurons in the $\mathrm{OB}$ of transgenic zebrafish. However, this serendipitous result prompted us to examine whether the lh $x 2 a$ promoter can be used for anatomical and functional analyses of these OB neurons, especially visualization of the secondary olfactory pathway from the $\mathrm{OB}$ to higher olfactory centers.

\section{lhx $2 a$ promoter drives transgene expression in mitral cells of the olfactory bulb}

To clearly visualize axonal trajectory of the OB neurons, we generated another transgenic zebrafish line in which gap-YFP is expressed under the control of the $\operatorname{lh} \times 2 a$ promoter (lhx2a:gap-YFP). gap-YFP is a yellow fluorescent protein tagged with a membrane-targeting palmitoylation signal of GAP43, enabling us to observe fine cellular morphology (Miyasaka et al., 2005; Sato et al., 2005). Careful observations of confocal optical sections revealed that gap-YFP-expressing OB neurons extended axonal processes into the ipsilateral telencephalon, some of which crossed the midline through the anterior commissure to reach the contralateral telencephalon (Fig. $1 D-D^{\prime \prime}$ ). These results imply that the $10 \mathrm{~kb}$ lh $x 2 \mathrm{a}$ promoter drives transgene expression in the $\mathrm{OB}$ output neurons.

To further characterize the $\mathrm{GFP}^{+} \mathrm{OB}$ neurons, we performed whole-mount double-fluorescence immunohistochemistry of 7-dpf lhx2a:GFP larvae with neuron type-specific marker antibodies. The granule cells, GABAergic inhibitory local interneurons, are located in the innermost portion of the OB (Fig. $2 A, A^{\prime}$ ). $\mathrm{GFP}^{+}$ neurons were found more peripheral to the $\mathrm{GABA}^{+}$granule cells without any profile of colocalization (Fig. $2 A^{\prime}$ ) and were apposed to the glomeruli labeled with an antibody against SV2, a presynaptic marker (Fig. 2C). Another type of $\mathrm{OB}$ interneuron is the juxtaglomerular cell, whose subpopulation expresses $\mathrm{TH}$. The $\mathrm{TH}^{+}$juxtaglomerular cells were distributed in a region that partly overlaps with that of $\mathrm{GFP}^{+}$neurons (Fig. $2 \mathrm{~B}$ ). However, a close examination of individual optical sections revealed that all the $\mathrm{GFP}^{+}$neurons were negative for TH (Fig. $2 B^{\prime}$ ). These results indicate that the $\mathrm{GFP}^{+} \mathrm{OB}$ neurons in lhx2a:GFP larvae are not the $\mathrm{GABA}^{+}$or
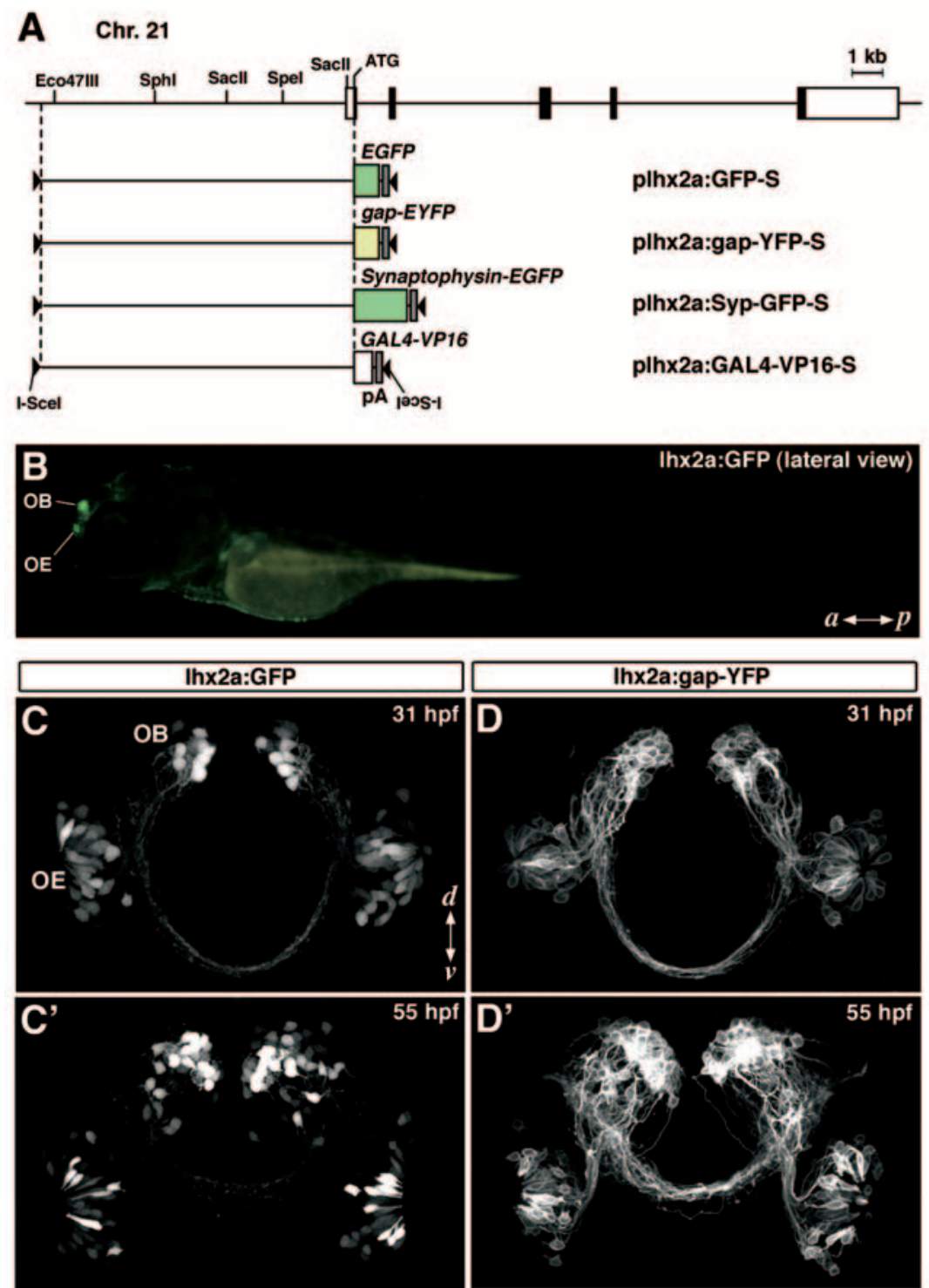

$55 \mathrm{hpf}$
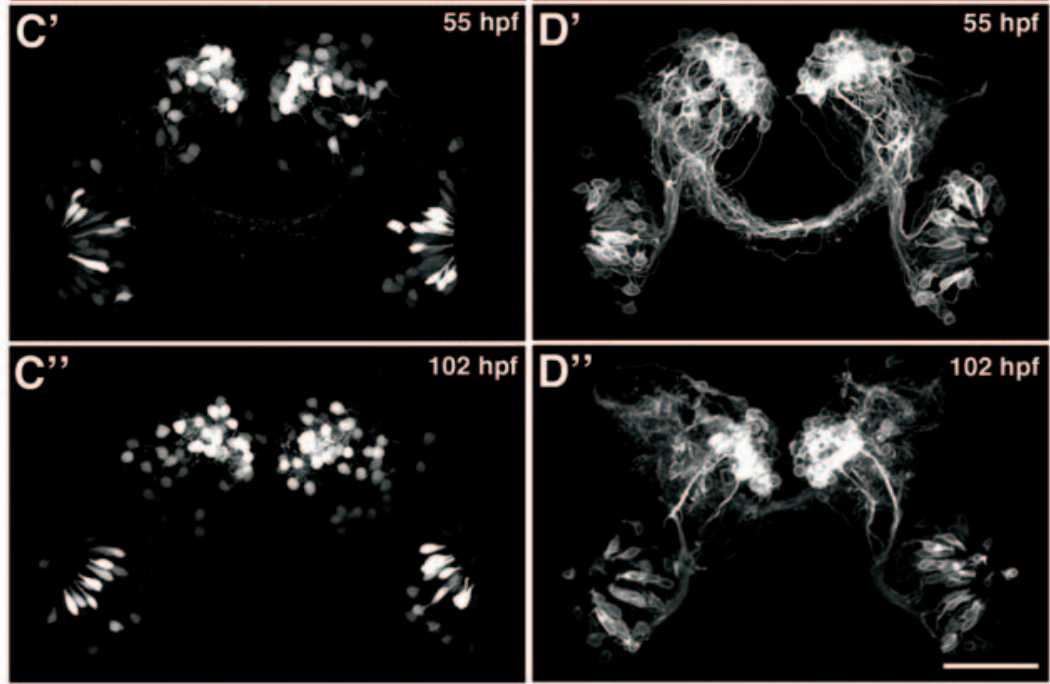

Figure 1. Generation of transgenic zebrafish with /hx2a promoter. $A$, Genomic organization of the /hx2a gene and structure of DNA constructs for generating transgenic fish. The black and white boxes (top) indicate the coding and noncoding regions of the Ihx2a gene exons, respectively. The $10 \mathrm{~kb}$ sequence upstream from the translation start site (ATG) is used to drive expression of EGFP, gap-EYFP, Synaptophysin-EGFP, and GAL4-VP16. pA, SV40 polyadenylation site; I-Scel, I-Scel meganuclease recognition site. $\boldsymbol{B}$, A fluorescence stereomicroscope image of a $3 \mathrm{dpf}$ Ihx2a:GFP larva viewed from lateral side. Anterior is to the left. $\mathbf{C}-\boldsymbol{C}^{\prime \prime}$, $\boldsymbol{D}-\boldsymbol{D}^{\prime \prime}$, Time-lapse imaging of Ihx2a:GFP (C-C“) and Ihx2a:gap-YFP (D-D') fish from day 1 to day 4 of development. Fish are shown in frontal views as confocal Z-stacked images. GFP-labeled cell bodies are observed not only in the OE but also in the OB of Ihx2a:GFP fish ( $\left(\boldsymbol{C}^{\prime \prime}\right)$. The expression of membrane-tethered YFP in Ihx2a:gap-YFP fish facilitates visualization of axonal processes $\left(D^{\left.-D^{\prime \prime}\right) .} a\right.$, Anterior; $p$, posterior; $d$, dorsal; $v$, ventral. Scale bar: (in $\left.\boldsymbol{D}^{\prime \prime}\right) \mathbf{C}-\mathbf{C}^{\prime \prime}$ and $\mathbf{D}-\boldsymbol{D}^{\prime \prime}, 50 \mu \mathrm{m}$.

$\mathrm{TH}^{+}$local interneurons, consistent with the above observation that they extended axons outside the OB (Fig. $1 D-D^{\prime \prime}$ ).

Adult $\mathrm{OB}$ contains a group of neuroendocrine cells, the terminal nerve cells, which express GnRH (Whitlock, 2004). During 

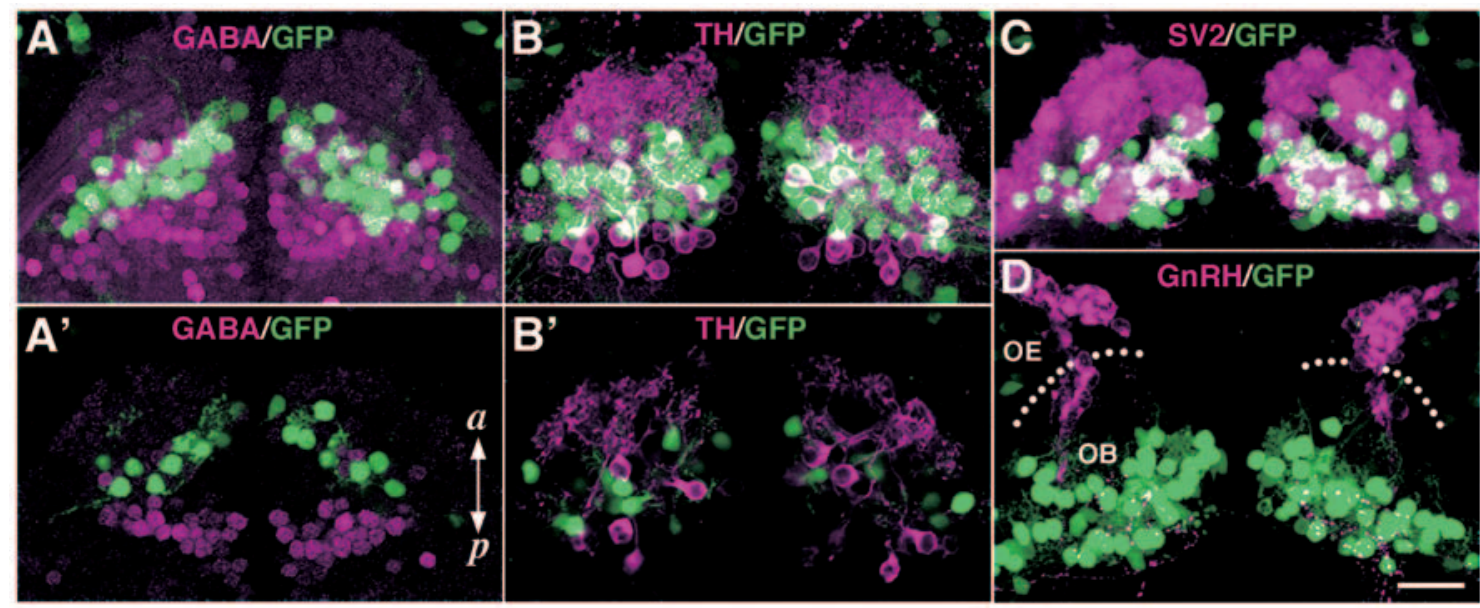

Figure 2. Characterization of labeled OB neurons in Ihx2a:GFP larvae. Whole-mount double-fluorescence labeling of 7-dpf Ihx2a:GFP larvae with anti-GFP antibody (green) and four marker antibodies (magenta). OBs are shown in dorsal views. $\boldsymbol{A}-\boldsymbol{D}$, Confocal Z-stacked images. $\boldsymbol{A}^{\prime}, \boldsymbol{B}^{\prime}$, Selected single-optical sections. $\boldsymbol{A}, \boldsymbol{A}^{\prime}, \boldsymbol{B}, \boldsymbol{B}^{\prime}$, Anti-GABA and TH antibodies were used to label granule cells and juxtaglomerular cells, respectively. GFP ${ }^{+} \mathrm{OB}$ neurons express neither $\mathrm{GABA}$ nor TH. $C, \mathrm{GFP}^{+} \mathrm{OB}$ neurons are apposed to glomeruli defined by staining with anti-SV2 antibody. $\boldsymbol{D}$, Terminal nerve cells that contain $\mathrm{GnRH}$ are arrayed along the olfactory axons and segregated from GFP ${ }^{+} \mathrm{OB}$ neurons. Dotted lines in $D$ indicate the anterior edge of the brain. $a$, Anterior; $p$, posterior. Scale bar, $20 \mu \mathrm{m}$.

development, the terminal nerve cells are born in the vicinity of the olfactory placode, migrate along the OSN axons to enter the ventral forebrain, and eventually populate the $\mathrm{OB}$ (Whitlock, 2004). At $7 \mathrm{dpf}$, the $\mathrm{GnRH}^{+}$terminal nerve cells were arrayed along the olfactory axons, some of which had entered the ventral forebrain. However, these terminal nerve cells were completely segregated from the $\mathrm{GFP}^{+} \mathrm{OB}$ neurons (Fig. 2D). Together, these results suggest that the $\mathrm{GFP}^{+} \mathrm{OB}$ neurons of lhx2a:GFP transgenic zebrafish are the output neurons, MCs.

\section{Molecular heterogeneity of mitral cells in the zebrafish olfactory bulb}

In 7-dpf zebrafish larvae, OB glomeruli were spatially segregated into five major clusters, which were clearly delineated by whole-mount immunolabeling with anti-SV2 antibody (Fig. $\left.3 A^{\prime}-C^{\prime}, G-I\right)$. We designated these clusters as the anteroventral, dorsal, posterior, lateral, and medial glomerular (avG, dG, pG, $1 \mathrm{G}$, and $\mathrm{mG}$, respectively) clusters (Fig. $3 J$ ), so that they reflect the previous designations for glomerular groups of 3-dpf larvae (Dynes and Ngai, 1998) and adult fish (Baier and Korsching, 1994). We previously demonstrated in OMP:lyn-RFP;TRPC2: gap-Venus double transgenic fish that the two distinct types of OSNs project their axons to spatially non-overlapping sets of glomeruli (Sato et al., 2005). OMP:lyn-RFP ${ }^{+}$ciliated OSNs expressing OR-type receptors project axons to the avG, $\mathrm{dG}$, and a subset of pG clusters (Fig. 3G,I,J), whereas TRPC2:gap-Venus ${ }^{+}$ microvillous OSNs expressing V2R-type receptors project axons predominantly to the $\mathrm{lG}$ cluster and partially to the avG and $\mathrm{pG}$ clusters (Fig. $3 \mathrm{H}, I, J$ ). In contrast, the $\mathrm{mG}$ cluster is innervated by neither OMP:lyn-RFP ${ }^{+}$nor TRPC2:gap-Venus ${ }^{+}$axons (Fig. $3 I, J)$, indicating the presence of at least three classes of glomeruli with distinct OSN axon innervations.

We examined the relationship between the distribution of Ihx2a:GFP ${ }^{+}$MCs and the glomerular classes defined by OSN axon innervations. The majority of $\operatorname{lh} \times 2 a: G F P^{+}$MCs were located adjacent to the $\mathrm{mG}$ cluster (Fig. $3 A^{\prime}$ ), which lacks the innervations by OMP:lyn-RFP ${ }^{+}$and TRPC2:gap-Venus ${ }^{+}$axons in the double transgenic larvae (Fig. 3I). Several lhx2a: $\mathrm{GFP}^{+} \mathrm{MCs}$ were also found in the proximity of other glomerular clusters (Fig. $3 A^{\prime}$ ), which receive inputs from either $O M P$ :
lyn-RFP ${ }^{+}$or TRPC2:gap-Venus ${ }^{+}$axons (Fig. 3I). These observations suggest that the $\ln \times 2 a: G F P^{+} \mathrm{OB}$ neurons are a spatially restricted subpopulation of MCs that presumably convey the odor information mainly represented in the $\mathrm{mG}$ cluster to higher olfactory centers.

Because only a subpopulation of MCs was labeled with the lhx 2 a promoter, we generated transgenic lines with other transcriptional promoters on the basis of the results of our literature research. Tbr2 is a T-box transcription factor expressed in MCs of the mouse OB (Kimura et al., 1999), whose orthologous gene $t b r 2 a$ is also found in the zebrafish OB (Mione et al., 2001). In tbr2a:DsRed transgenic line, DsRed protein was expressed in a subpopulation of MCs under the control of $5 \mathrm{~kb}$ upstream regulatory region of the zebrafish tbr2a gene (Fig. 3B; supplemental Fig. 3, available at www.jneurosci.org as supplemental material). The $t b r 2 a: D s R e d^{+}$MCs were preferentially located surrounding the $\mathrm{mG}$ and avG clusters (Fig. $3 B^{\prime}$ ). vglut $2 a$ is a zebrafish orthologue of the mammalian gene encoding the vesicular glutamate transporter Vglut2 (Higashijima et al., 2004; Smear et al., 2007). We analyzed two zebrafish lines, vglut2a:DsRed and vglut2a: GFP, with bacterial artificial chromosome (BAC) transgenes which harbor DsRed and GFP, respectively. DsRed (or GFP) was expressed in a subpopulation of MCs (Fig. 3C,E; supplemental Fig. 3, available at www.jneurosci.org as supplemental material), which were predominantly situated posteriorly adjacent to the $\mathrm{dG}$ cluster (Fig. $3 C^{\prime}$ ). These results indicate that the three transgenic lines with different promoters (lh $\times 2 a, t b r 2 a, v g l u t 2 a)$ exhibit distinct distributions of the labeled MCs in the OB (Fig. 3J).

To further examine the relationship among the three subpopulations of labeled MCs, we generated double transgenic fish with three combinations: lhx2a:GFP;tbr2a:DsRed, tbr2a:DsRed; vglut2a:GFP, and vglut2a:DsRed;lhx2a:GFP. Surprisingly, nearly all the MCs did not coexpress the two fluorescent proteins in each double transgenic line, indicating that they are virtually nonoverlapping subpopulations of MCs (Fig. 3D-F). These results suggest that MCs exhibit molecular heterogeneity and each subpopulation of MCs displays distinct spatial distribution with remarkable correlations to the glomerular classes defined by the OSN axon innervations. 

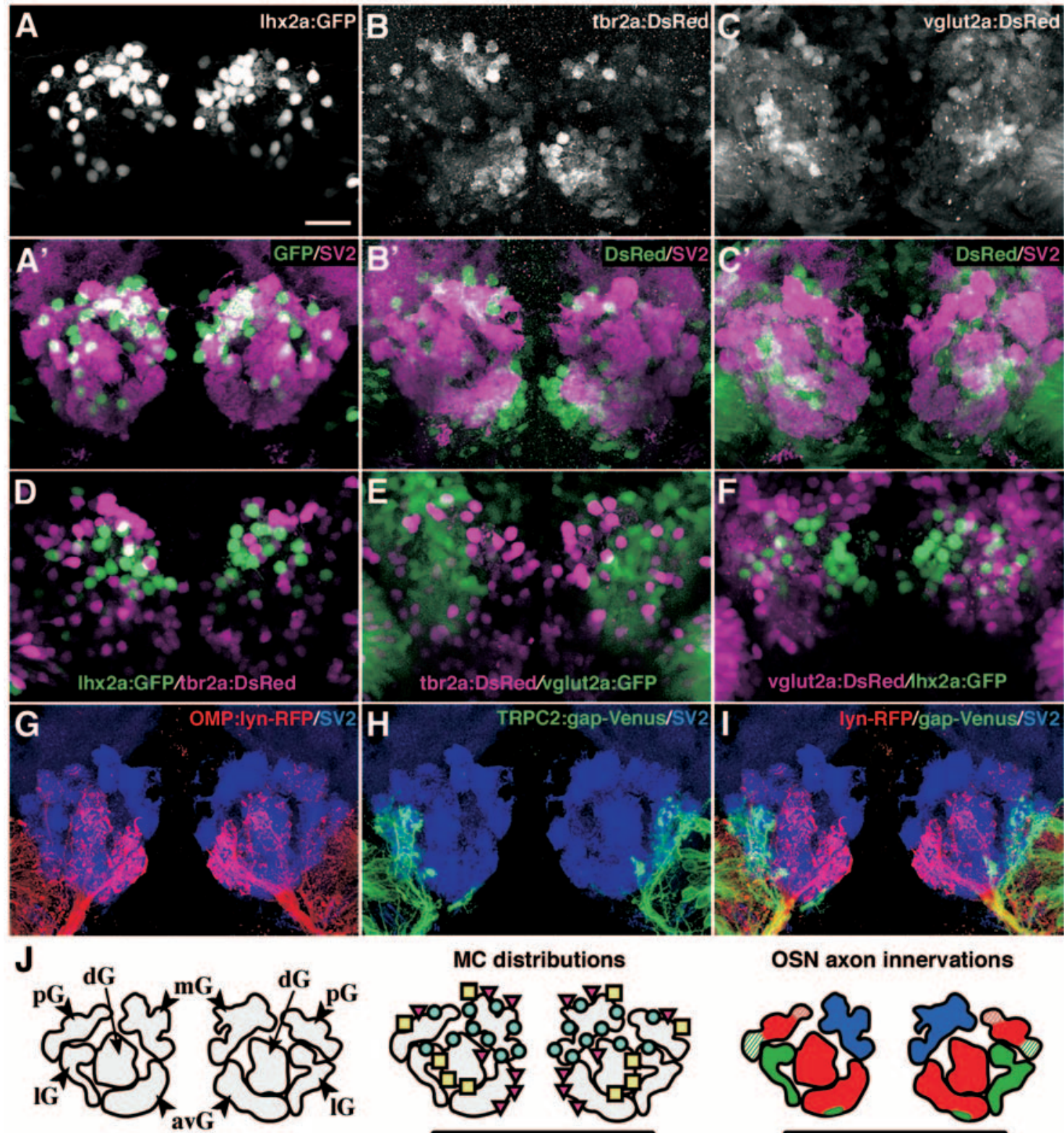

MC distributions

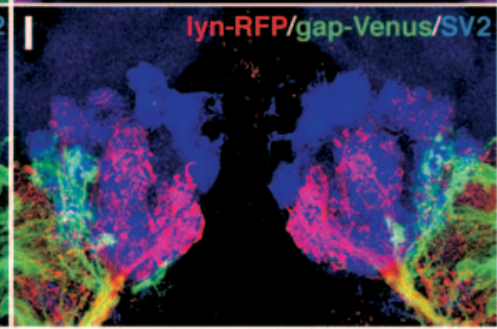

$\mathbf{J}$
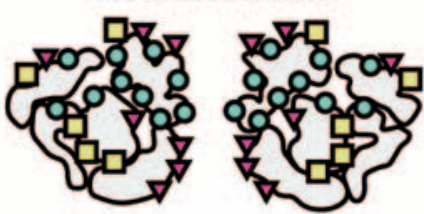

OSN axon innervations

\section{Glomerular clusters (frontal view)}

\begin{tabular}{|c|c|}
\hline $\begin{array}{c}\text { Ihx } 2 a^{t g} \\
\text { tbr2atg } \\
\text { vglut2 } 2 a^{t g}\end{array}$ & $\begin{array}{l}0 \\
\nabla \\
\square\end{array}$ \\
\hline
\end{tabular}

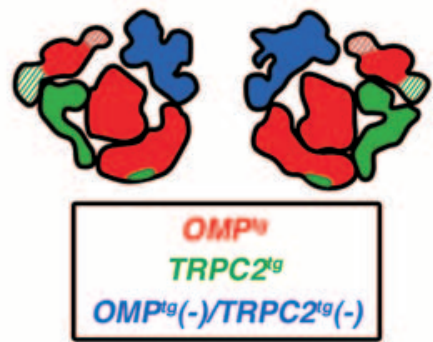

Figure 3. Heterogeneity of mitral cells revealed in three transgenic lines with different promoters. $A-C, A^{\prime}-C^{\prime}$, Whole-mount double-fluorescence labeling of 7-dpf lhx2a:GFP, tbr2a:DsRed, and vglut2a:DsRed transgenic larvae with antibodies against SV2 (magenta in $\boldsymbol{A}^{\prime}-\boldsymbol{C}^{\prime}$ ) and GFP ( $\boldsymbol{A}$; green in $\left.\boldsymbol{A}^{\prime}\right)$ or DsRed ( $\boldsymbol{B}$, $\boldsymbol{C}$; green in $\boldsymbol{B}^{\prime}$ and $\boldsymbol{C}$ ). Larvae are shown in frontal views as confocal Z-stacked images. Ihx2a:GFP ${ }^{+}$, tbr2a:DsRed ${ }^{+}$, and vglut2a:DsRed ${ }^{+} \mathrm{MCs}$ are predominantly apposed to the $\mathrm{mG}, \mathrm{mG}$ and avG, and dG clusters, respectively. $\boldsymbol{D}-\boldsymbol{F}$, Nearly all GFP ${ }^{+}$MCs (green) do not coexpress DsRed (magenta) in each double transgenic fish with three combinations, Ihx2a:GFP;tbr2a:DsRed (D), tbr2a:DsRed;vglut2a:GFP (E), and vglut2a:DsRed;/hx2a:GFP (F). G-I, Whole-mount triplefluorescence labeling of a 7-dpf double transgenic larva carrying OMP:Iyn-RFP and TRPC2:gap-Venus transgenes with antibodies against lyn-RFP (red), gap-Venus (green), and SV2 (blue). J, Schematic drawings representing the glomerular clusters (left), MC distributions (middle), and OSN axon innervations (right). Scale bar, $20 \mu \mathrm{m}$.

Axon projection of $\operatorname{lh} \times 2 a^{t g+}$ mitral cells in zebrafish larvae Because the transgenes were expressed not only in the MCs but also in various types of forebrain neurons in the tbr $2 \mathrm{a}$ and vglut $2 \mathrm{a}$ transgenic lines (supplemental Fig. 3, available at www. jneurosci.org as supplemental material), it was difficult to trace the axon trajectories selectively from these two populations of MCs. In the lhx2a transgenic lines, in contrast, a subpopulation of MCs in the OB were the only cell type expressing the fluorescent proteins within the brain (Fig. $4 \mathrm{~B}$ ), allowing us to observe a de- tailed pattern of $\mathrm{MC}$ axon projection from the $\mathrm{OB}$ to higher olfactory centers. In $7 \mathrm{dpf}$ larvae of the lhx2a:gap-YFP line, the labeled MCs extended axons from the OB into the telencephalon, forming two loosely fasciculated bundles, presumptive lateral and medial olfactory tracts (LOT and MOT) (Fig. 4C). These MC axons elaborated many branches within bilaterally symmetric fields in the left and right telencephalic hemispheres, which were interconnected by the axonal processes through the anterior commissure (Fig. 4C). A population of MC axons emerged from 

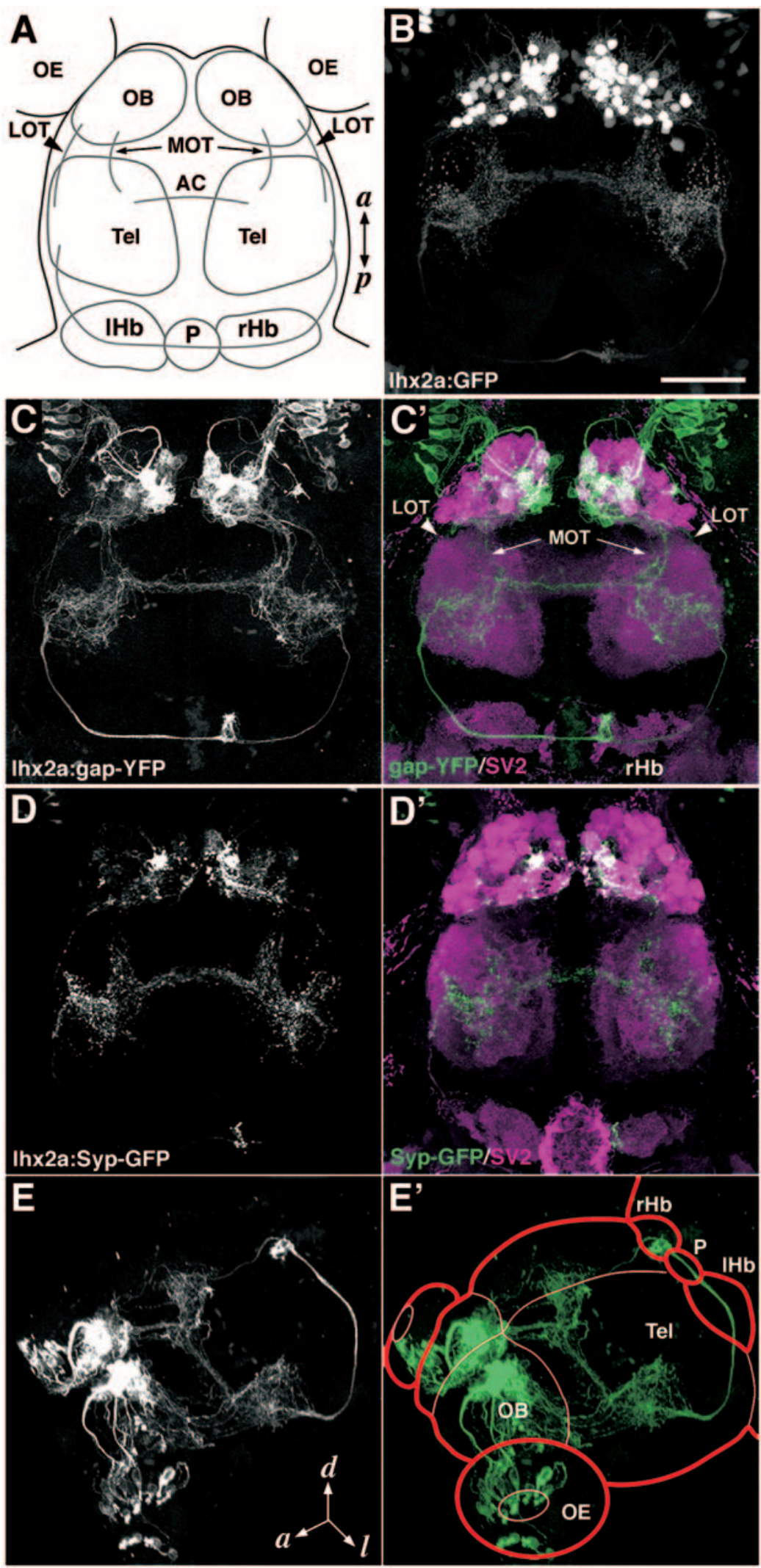

Figure 4. Axon projection pattern of $/ h \times 2 a^{\text {tg }}+$ mitral cells in 7-dpf larvae. $A$, Diagram representing the dorsal view of zebrafish forebrain at $7 \mathrm{dpf}$. $\boldsymbol{B}$, GFP fluorescence of a Ihx2a:GFP larva is shown in a dorsal view. $\boldsymbol{C}, \boldsymbol{C}^{\prime}$, A Ihx2a:gap-YFP larva double-labeled with antibodies against gap-YFP ( $\boldsymbol{C}$; green in $\boldsymbol{C}$ ) and SV2 (magenta in $\boldsymbol{C}$ ). The MC axons extend into the telencephalon via presumptive $\mathrm{LOT}$ and $\mathrm{MOT}$, arborize in bilaterally symmetric fields of the telencephalon, and cross the midline through the anterior commissure. Some of the axons further extend posteriorly to reach the right habenula. $\boldsymbol{D}, \boldsymbol{D}^{\prime}, \mathrm{A}$ Ihx2a:Syp-GFP larva double-labeled with antibodies against Syp-GFP ( $\boldsymbol{D}$; green in $\boldsymbol{D}$ ) and SV2 (magenta in $\boldsymbol{D}$ ). Presynaptic sites are labeled with Synaptophysin-EGFP (Syp-GFP) fusion protein. Punctate signals of Syp-GFP are observed in bilateral fields of telencephalon and the right habenula. the telencephalic arborization, formed a tight fascicle in each hemisphere, and further extended posteriorly (Fig. 4C). The fasciculated axons then abruptly made a dorsal turn, reached the dorsal surface of the epithalamus, and crossed the midline through the habenular commissure (Fig. $\left.4 C, C^{\prime}, E, E^{\prime}\right)$. Surprisingly, axon terminallike structures were present only in the right hemisphere of the epithalamus, the right habenula (Fig. $4 C, C^{\prime}$ ). In 3 dpf larvae, we occasionally observed an extremely small population of axons extending posteriorly through the ventral telencephalon from the OB directly to the hypothalamus (supplemental Fig. 4, available at www.jneurosci.org as supplemental material). However, it was difficult to detect such axonal fibers at $7 \mathrm{dpf}$ (data not shown), possibly due to their low fluorescence intensity and deep position in the brain.

To precisely locate the axon terminals of $\operatorname{lh} \times 2 a^{\operatorname{tg}+}$ MCs, we generated another transgenic line, lhx2a:Syp-GFP, in which synaptic vesicles are labeled with Synaptophysin-EGFP fusion protein (SypGFP). Punctate Syp-GFP signals were clearly observed in the right habenula as well as the bilateral telencephalic fields of 7-dpf lhx2a:Syp-GFP larvae (Fig. 4D, $D^{\prime}$ ), confirming that the putative axon terminals of MCs in the lhx2a:gap-YFP line actually contained synaptic vesicles. A strongly fluorescent glomerulus within the $\mathrm{mG}$ cluster of the $\mathrm{OB}$ contained the presynaptic axon terminals of the $\ln \times 2 a^{\operatorname{tg}+}$ OSNs (Fig. 4, compare $C^{\prime}, D^{\prime}$ ).

We further characterized the left-right asymmetric habenular projection from the $\mathrm{OB}$ by immunolabeling the habenulae of lhx2a:gap-YFP larvae with several marker antibodies. Go was present in the entire membranous structure of the habenulae (Fig. 5B), allowing us to observe the somata of habenular neurons surrounding a densely labeled central neuropil domain. The central neuropil domain was composed of SV $2^{+}$presynaptic terminals of afferent fibers (Fig. 5A) and MAP $2^{+}$dendritic arborizations of the habenular neurons (Fig. 5C). The gap-YFP ${ }^{+} \mathrm{MC}$ axons terminated restrictedly to the medial portion of the central neuropil domain in

$\longleftarrow$

Strong signals in a few glomeruli contain the axon terminals of the $/ h \times 2 a^{t g+}$ OSNs. $\boldsymbol{E}, \boldsymbol{E}^{\prime}$, Three-dimensional reconstruction of gap-YFP-labeled brain structures viewed from anterodorsolateral side. Tel, Telencephalon; AC, anterior commissure; $\mathrm{IHb}$, left habenula; $\mathrm{rHb}$, right habenula; $\mathrm{P}$, pineal organ; $a$, anterior; $p$, posterior; $d$, dorsal; I, lateral. Scale bar: (in $\boldsymbol{B}) \boldsymbol{B}-\boldsymbol{D}$, $50 \mu \mathrm{m}$. 
the right habenula (Fig. $5 A^{\prime}-C^{\prime}$ ). OCAM is a cell adhesion molecule that is expressed in a zone-specific manner in the mouse olfactory system (Yoshihara et al., 1997). We found that zebrafish OCAM is expressed in the habenulae in a compartment-specific manner. In the right habenula, the lateral compartment of the central neuropil domain was strongly OCAM-positive, whereas the medial compartment showed no or little, if any, immunoreactivity for OCAM (Fig. 5D). The gap-YFP ${ }^{+} \mathrm{MC}$ axon terminals were observed in the medial neuropil compartment (Fig. $5 D^{\prime}$ ), suggesting that $\operatorname{lh} \times 2 a^{\operatorname{tg}+}$ MCs specifically innervate the OCAMnegative population of neurons in the right habenula. We encountered a small proportion of larvae exhibiting reversed laterality of the habenulae where the leftright asymmetric pattern of OCAM expression was inversed. In these larvae, $\mathrm{MC}$ axons innervated the medial neuropil compartment devoid of OCAM immunoreactivity in the left habenula, indicating a close correlation between the habenular laterality and the MC axon innervation (data not shown).

\section{Visualization of mitral cells at single-cell resolution}

How do individual MCs innervating particular glomeruli project their axons to higher olfactory centers? To address this question, we used a genetic mosaic labeling technique: a mixture of GAL4VP16 driver and UAS:gap-YFP responder plasmids was injected into 1- to 4-cell-stage embryos, resulting in mosaic and transient expression of gap-YFP at various frequencies ranging from single cells to large populations of cells. Fine cellular morphology, including dendritic arborization and axon trajectory, of singlelabeled MCs were examined at $4 \mathrm{dpf}$ (data not shown) and again at $7 \mathrm{dpf}$ (Fig. $\left.6 B_{1}-B_{4}, C_{1}-F_{1}\right)$ in living larvae. Subsequently, their target glomeruli were identified by immunolabeling with antibodies against gap-YFP and SV2 (Fig. $6 C_{0}-F_{0}$ ).

Using $l h x 2 a$ promoter to drive GAL4-VP16 expression, single-cell images were obtained at high resolution for $27 \mathrm{MCs}$ in 334 larvae analyzed. We first observed the dendritic morphology and glomerular innervation of individual MCs. All the $27 \mathrm{MCs}$ exhibited the uni-dendritic morphology (Fig. $6 B_{1}-B_{3}$ ). The majority of labeled MCs had a short primary dendrite with either a large (Fig. $6 B_{1}$ ) or small (Fig. $6 B_{2}$ ) tuft innervating a glomerulus close to the soma (24 of $27 \mathrm{MCs}, 89 \%$ ), while other three MCs (11\%) extended a relatively long dendrite reaching a distant glomerulus (Fig. $6 B_{3}$ ). Consistent with the distribution of labeled MCs in lhx2a:GFP transgenic line (Fig. $3 A, A^{\prime}$ ), most of the single-labeled MCs exhibited dendritic innervations to one of the six discrete glomeruli (designated as $\mathrm{mG1}$ to $\mathrm{mG}$ ) in the $\mathrm{mG}$ cluster (24 of 27 MCs, 89\%) (Table 1, Fig. 6A).

We next analyzed the patterns of axon projections of singlelabeled MCs. There was no significant difference in the overall axon trajectories of individual MCs between 4 and $7 \mathrm{dpf}$, although pruning of a few processes was observed on rare occasions during this developmental period (data not shown). The vast majority of MCs connecting a dendrite with the $\mathrm{mG}$ cluster innervated the telencephalon bilaterally via the anterior commis- sure (23 of $24 \mathrm{MCs}, 96 \%$ ) and further projected axons to the right habenula (20 of 24 MCs, 83\%) (Table 1). Representative images of the bulbo-habenular projection are shown for two mG1innervating MCs (mG1-MCs) (Fig. 6C $C_{1}, C_{2}$ ) and three mG6MCs (Fig. $6 D_{1}-D_{3}$ ). These $\mathrm{MC}$ axons entered the ipsilateral telencephalon to make several branches, crossed the midline through the anterior commissure to make branches again in the contralateral telencephalon, and also reached the right habenula. These observations suggest that individual MCs are capable of sending odor information simultaneously to multiple target regions in the forebrain: both hemispheres of the telencephalon and the habenular nucleus. For 1 of the $5 \mathrm{mG} 1-\mathrm{MCs}$ (Fig. $6 C_{3}$ ) and 1 of the 12 mG6-MCs (data not shown), however, the axons did not project to the right habenula and showed more complicated branching patterns in the telencephalon when compared with other mG1- and mG6-MCs (Fig. $6 C_{1}, C_{2}, D_{1}-D_{3}$ ). This result indicates that MCs innervating the same glomerulus do not always display a stereotypic pattern of axon projection.

In addition, we noticed that even when MCs innervating the same glomerulus project axons to the same target regions, their routes are variable (Fig. $6 D_{1}-D_{3}$ ). For example, 7 of the 11 mG6MCs projected axons to the right habenula from the contralateral telencephalon after crossing the midline (termed as "S-shape" trajectory) (Fig. $6 D_{1}, D_{2}$ ), whereas the other $4 \mathrm{mG} 6-\mathrm{MCs}$ projected axons to the right habenula from the ipsilateral telencephalon (termed as "E-shape" trajectory) (Fig. 6 $D_{3}$ ). There was no correlation between the origin of MCs in either the left or right $\mathrm{OB}$ and their axonal routes reaching the right habenula. Among the $11 \mathrm{mG6}-\mathrm{MCs}$, we observed five S-shape and three E-shape trajectories from the left $\mathrm{OB}$ and two S-shape and one E-shape trajectories from the right $\mathrm{OB}$. Intriguingly, many of the $\mathrm{mG}-\mathrm{MC}$ axons crossed the midline twice, first through the anterior commissure and then through the habenular commissure (Fig. $\left.6 C_{1}, C_{2}, D_{2}, D_{3}\right)$. These observations imply that the pathfinding of 

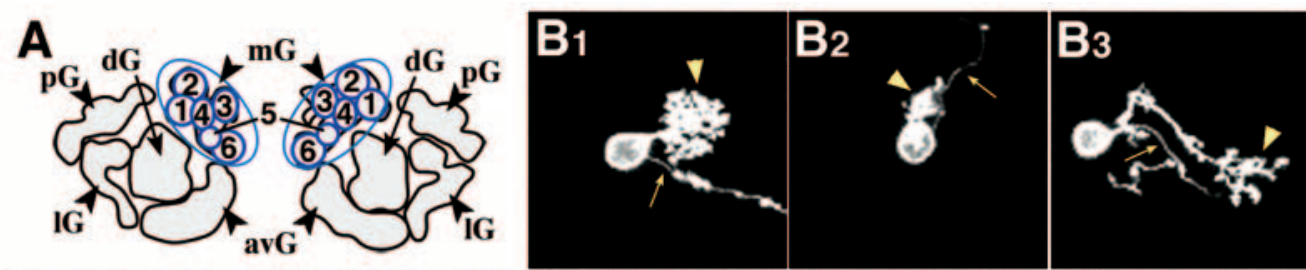

\section{$\mathrm{B} 4$}
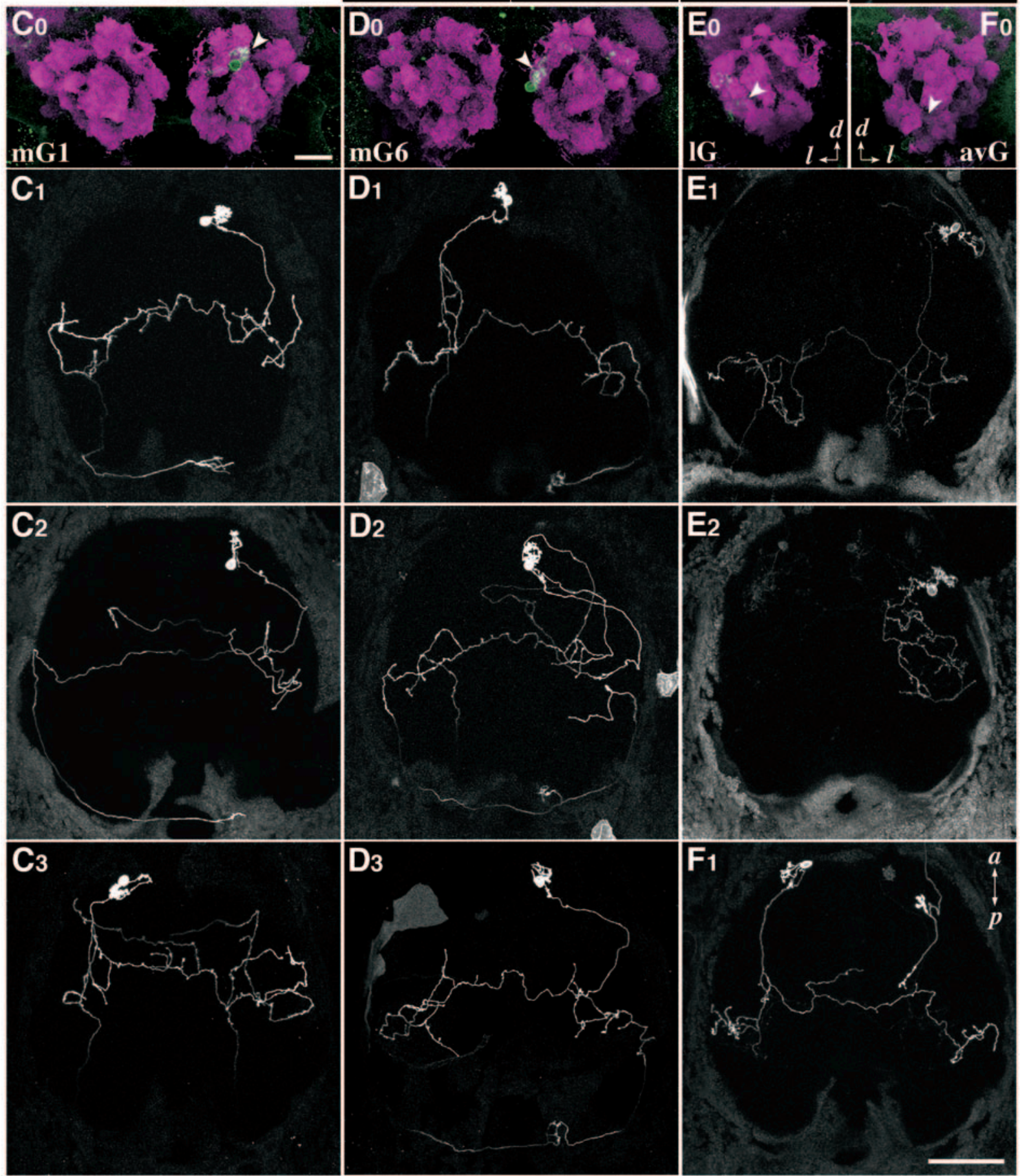

Figure 6. Genetic visualization of single mitral cells. $\boldsymbol{B}-\boldsymbol{F}$, Single-cell labeling was performed by injecting a mixture of two plasmids, plhx2a:GAL4-VP16-S and pUAS:gap-YFP ( $\left.\boldsymbol{B}_{\mathbf{1}}-\boldsymbol{B}_{3}, \boldsymbol{C}-\boldsymbol{E}\right)$, or ptbr2a:GAL4-VP16 and pUAS:gap-YFP $\left(\boldsymbol{B}_{4}, \boldsymbol{F}\right)$, into 1-4 cell-stage embryos. The dendritic innervation pattern and the axon trajectory were examined at 7 dpf. $\boldsymbol{A}$, Schematic representation of $0 B$ glomerular clusters and $\mathrm{mG}$ numbering. $\boldsymbol{B}_{1}-\boldsymbol{B}_{4}$, Typical dendritic morphologies of labeled MCs. Arrows and arrowheads denote the axons and the dendritic tufts, respectively. $\boldsymbol{C}_{\boldsymbol{0}}-\boldsymbol{F}_{\boldsymbol{0}}$, Examples of glomerular innervation patterns of four MC classes. $\boldsymbol{C}_{1}-\boldsymbol{C}_{3}$, Axon projection patterns of mG1-MCs in three different larvae. $\boldsymbol{D}_{1}-\boldsymbol{D}_{3}$, Axon projection patterns of $\mathrm{mG} 6-\mathrm{MCs}$ in three different larvae. $\boldsymbol{E}_{1}, \boldsymbol{E}_{2}$, Axon projection patterns of $\mathrm{G}-\mathrm{MC}$ in two different larvae. $\boldsymbol{F}_{1}$, An axon projection pattern of avG-MC in a larva. $\boldsymbol{C}_{0}-\boldsymbol{F}_{0}$, Frontal views with dorsal to the top. $\boldsymbol{C}_{1}-\boldsymbol{F}_{1}$, Dorsal views with anterior to the top. $a$, Anterior; $p$, posterior; $d$, dorsal; I, lateral. Scale bar: (in $\boldsymbol{B}_{4}$ ) $\boldsymbol{B}_{1}-\boldsymbol{B}_{4}, 10 \mu \mathrm{m}$; (in $\boldsymbol{C}_{0}$ ) $\boldsymbol{C}_{0}-\boldsymbol{F}_{0}, 20 \mu \mathrm{m}$; (in $\boldsymbol{F}_{7}$ ), $\boldsymbol{C}_{1}-\boldsymbol{F}_{1}, 50 \mu \mathrm{m}$. 
MC axons might be regulated by a peculiar guidance mechanism involving in part the permissive routes to the final destinations.

We encountered 3 single-labeled MCs that innervate glomeruli outside the $\mathrm{mG}$ cluster ( 3 of $27 \mathrm{MCs}, 11 \%$ ) with the $l h \times 2 a$ promoter. An MC innervating a glomerulus within the $\mathrm{pG}$ cluster, which locates laterally adjacent to the $\mathrm{mG}$ cluster, displayed a trajectory similar to the E-shape observed in $\mathrm{mG}-\mathrm{MCs}$ (data not shown). However, two MCs innervating a glomerulus within the $1 \mathrm{G}$ cluster showed different patterns of axon projection. One lG-MC innervated a posterior field in the telencephalon bilaterally (Fig. 6E $E_{1}$ ) and the other $1 G$-MC projected an axon only ipsilaterally to the anterior telencephalon (Fig. $6 E_{2}$ ). However, no projection to the habenula was observed for these $1 \mathrm{G}-\mathrm{MCs}$ (Fig. $\left.6 E_{1}, E_{2}\right)$.

To label MCs that innervate glomeruli outside the $\mathrm{mG}$ cluster more efficiently, we used the tbr2a promoter as a GAL4-VP16 driver. Five single-labeled MCs (one mG4-, one $1 \mathrm{G}-$, one $\mathrm{dG}-$, and two avGMCs) were obtained from 106 larvae analyzed (Table 1). Four MCs showed unidendritic morphology, whereas the remaining one possessed multiple dendrites innervating the same region (Fig. $\left.6 B_{4}\right)$. The $\mathrm{mG} 4-\mathrm{MC}$ labeled with the $t b r 2 a$ promoter exhibited axon projection to the right habenula in the S-shape trajectory as observed for mG-MCs labeled with the $l h \times 2 a$ promoter. All the other four MCs displayed axon projections to the bilateral telencephalon without innervating the habenula (Table 1, Fig. $6 F_{1}$ ). Thus, MCs innervating glomeruli outside the $\mathrm{mG}$ cluster tended to display no habenular projection (six of seven MCs labeled with either the $l h x 2 a$ or $t b r 2 a$ promoter).

\section{Preserved axon projection of $l h x 2 a^{t g+}$ mitral cells in adult zebrafish}

We finally examined whether the axon projection pattern of lh $\times 2 a^{\text {tg+ }}$ MCs observed at larval stage is preserved in adult fish. A whole-mount lateral view of lhx2a:gap-YFP adult brain showed the presence of intense fluorescence in the $\mathrm{OB}$ and the telencephalon with no fluorescence in the midbrain and hindbrain (Fig. $7 A, A^{\prime}$ ). A whole-mount ventral view of the forebrain (Fig. $\left.7 C, C^{\prime}\right)$ and coronal sections through the anterior telencephalon (Fig. $7 D, E$ ) revealed that gap-YFP ${ }^{+}$axons exiting the OB run through the LOT and MOT and cross the midline through the dorsal part of the anterior commissure. At the posterior levels to the anterior commissure, numerous gap-YFP ${ }^{+}$fibers were found in the ventrolateral portion of the dorsal telencephalic area (Fig. $7 F$ ). It has been reported that two major nuclei in the dorsal telencephalic area [lateral zone of dorsal telencephalic area (Dl), posterior zone of dorsal telencephalic area (Dp)] can be distinguished by the difference of NPY immunoreactivity: the Dl contains numerous $\mathrm{NPY}^{+}$fibers, whereas the Dp receives sparse innervation of $\mathrm{NPY}^{+}$fibers (Castro et al., 2006). Double labeling for gap-YFP and NPY revealed that the region occupied with abundant gap-YFP ${ }^{+}$fibers corresponds to the Dp (Fig. 7F). A whole-mount dorsal view clearly revealed that the asymmetric projection from the $\mathrm{OB}$ to the right habenula was preserved also in the adult brain (Fig. $7 B, B^{\prime}$ ). We confirmed that the gap-YFP ${ }^{+}$ terminal-like structures were located within SV $2^{+}$neuropils in the right habenula (Fig. $7 G$ ). These observations indicate that the pattern of $\operatorname{lh} \times 2 a^{t g+} \mathrm{MC}$ axon projection is established during larval stages and maintained into adulthood.

\section{Discussion}

MCs are the principal output neurons in the OB, which relay odor information from OSNs to higher olfactory centers. Here we visualized the trajectory of MC axons in zebrafish at both population and single-cell levels using transgenesis with fluorescent proteins and obtained new findings as follows. (1) MCs are heterogeneous with respect to transgene expression profiles and spatial distributions. (2) Individual MCs project axons to multiple target regions in the forebrain. (3) MCs innervating different glomerular clusters tend to send axons to different, but partly overlapping, target regions. (4) MCs innervating the same glomerulus do not necessarily display the same axon trajectory. (5) MCs innervating different glomeruli within the $\mathrm{mG}$ cluster display axon convergence to the common target regions. (6) A large subpopulation of MCs innervating the $\mathrm{mG}$ cluster project axons directly and asymmetrically to the right habenula.

\section{Heterogeneity of mitral cells}

We found that three different transcriptional promoters (lh $x 2 a$, tbr2a, vglut2a) can drive transgene expression in distinct subpopulations of MCs, although their expression patterns do not necessarily recapitulate those of endogenous genes. This observation indicates that MCs are heterogeneous, comprising at least three different subpopulations. Individual MC subpopulations 

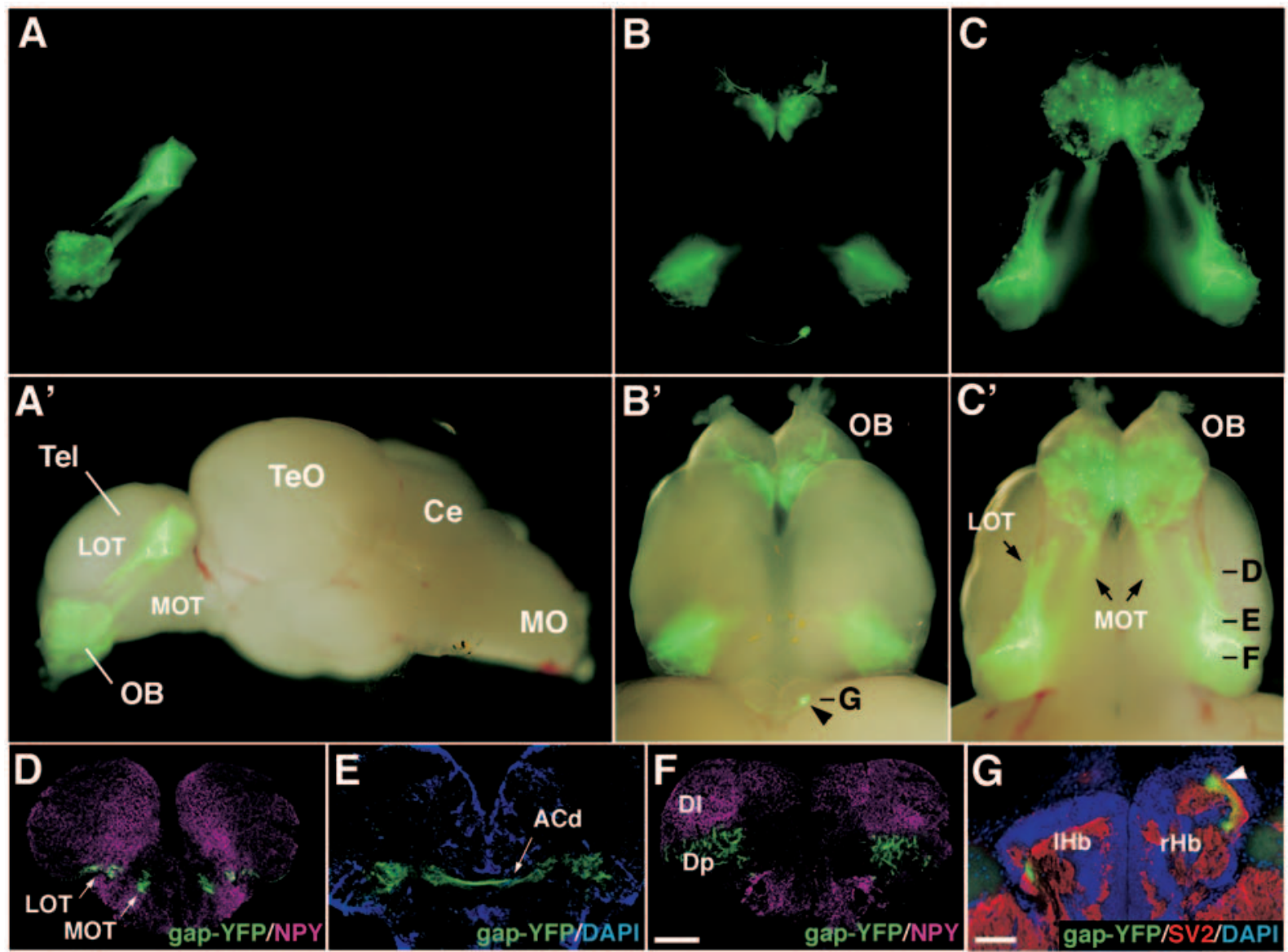

Figure 7. Preserved axon projection pattern of $/ h \times 2 a^{t g+}$ mitral cells in adult fish. $\boldsymbol{A}-\boldsymbol{C}, \boldsymbol{A}^{\prime}-\boldsymbol{C}^{\prime}$, A whole-mount brain from lhx2a:gap-YFP adult fish is shown with lateral $(\boldsymbol{A}, \boldsymbol{A})$, dorsal ( $\left.\boldsymbol{B}, \boldsymbol{B}\right)$ and ventral $\left(\boldsymbol{C}, C^{\prime}\right)$ views. $A-C$, Fluorescence images. $A^{\prime}-C^{\prime}$, Fluorescence superimposed on bright-field images. $\boldsymbol{D}-\mathbf{G}$, Coronal sections through the forebrain are immunostained with antibodies against gap-YFP (green), NPY, (magenta in $\boldsymbol{D}$ and $\boldsymbol{F}$ ), and SV2 (red in $\boldsymbol{G}$ ). Nuclear staining with DAPI (blue) is shown in $\boldsymbol{E}$ and $\boldsymbol{G}$. Approximate positions of individual sections are indicated in $\boldsymbol{B}^{\prime}$ and $\boldsymbol{C}^{\prime}$. Arrowheads in $\boldsymbol{B}^{\prime}$ and $\mathbf{G}$ denote the gap-YFP ${ }^{+}$axon terminals in the right habenula. Tel, Telencephalon; Te0, tectum opticum; Ce, cerebellum; M0, medulla oblongata; $A C d$, dorsal part of anterior commissure; $\mathbf{H b}$, left habenula; $\mathrm{rHb}$, right habenula. Scale bar: in $\boldsymbol{F}, 200 \mu \mathrm{m}$ for $\mathbf{D}-\boldsymbol{F}$; in $\mathbf{G}, 50 \mu \mathrm{m}$.

would have different gene expression profiles that are required for their characteristic dendritic innervations and axonal trajectories, leading to possible distinct functions. The three MC subpopulations exhibit distinct spatial distributions that are closely related to the odor map in the OB. Bile salts, putative social pheromones, are supposed to bind OR-type olfactory receptors in ciliated OSNs and activate glomeruli in the $\mathrm{dG}$ and avG clusters (Friedrich and Korsching, 1998; Li et al., 2005; Sato et al., 2005). We found that the majority of $v g l u t 2 a^{\text {tg+ }}$ MCs and a subset of $t b r 2 a^{\text {tg }}{ }^{2}$ MCs are located in the vicinity of the $\mathrm{dG}$ and avG clusters, respectively. Therefore, it is likely that these MCs may transmit the social information mediated by bile salts to higher olfactory centers. Amino acids, potent feeding cues, are mainly received by V2R-type olfactory receptors in microvillous OSNs and activate glomeruli in the lateral chain (1G cluster) (Friedrich and Korsching, 1997, 1998; Speca et al., 1999; Li et al., 2005; Sato et al., 2005). In this study, we observed only a few labeled MCs in the close proximity of the $\mathrm{lG}$ cluster in the three transgenic lines, suggesting the existence of another subpopulation of MCs predominantly conveying the amino acid information from the $\mathrm{lG}$ cluster to higher olfactory centers. It remains unknown what kinds of odorants activate and which types of OSNs innervate the $\mathrm{mG}$ cluster, to which the majority of $l h x 2 a^{\text {tgt }} \mathrm{MCs}$ and a subset of tbr2 $a^{t g+}$ MCs are apposed. Zebrafish has two other families of olfactory receptors: six V1R-type receptors (also known as ORAs)
(Saraiva and Korsching, 2007) and $\sim 100$ trace amine-associated receptors (TAARs) (Hashiguchi and Nishida, 2007). In addition, teleost fishes including zebrafish have the third type of OSNs, called crypt cells, with unknown functions (Hansen and Zeiske, 1998). Therefore, we speculate that the crypt cells may express V1R-type receptors or TAARs, project axons to the $\mathrm{mG}$ cluster, and mediate a unique olfactory response.

In addition to MCs, teleost fishes have another type of $\mathrm{OB}$ output neuron, the ruffed cell (Kosaka and Hama, 1979; Kosaka, 1980 ), although it constitutes only a minor population (e.g., < $5 \%$ of total OB output neurons in zebrafish) (Fuller and Byrd, 2005). The ruffed cells, although similar in size and localization to MCs, display a characteristic morphology having substantial membranous protrusions (ruffs) at the initial segment of the axons. In our Golgi-like single-cell labeling analysis, the labeled $\mathrm{OB}$ output neurons did not exhibit such axonal ruffs at $7 \mathrm{dpf}$. It is thus likely that they are the MCs, although we cannot exclude a possibility that the axonal ruff structure might be established during later developmental stages. Further studies are now in progress to label single OB output neurons in the adult zebrafish.

\section{Odor information transfer from the olfactory bulb to higher} brain centers

It has been long believed that MCs in teleost fishes typically have multiple primary dendrites innervating multiple glomeruli 
(Dryer and Graziadei, 1994). However, a recent study (Fuller et al., 2006) reported a revised description of zebrafish MC morphology in which the majority of retrogradely labeled MCs have a single primary dendrite with a discrete tuft and a smaller proportion of MCs showing the multidendritic morphology also innervates a single glomerulus. This notion was supported by the present result that the vast majority of genetically labeled single MCs (31 of $32 \mathrm{MCs}$ ) showed the uni-dendritic morphology, innervating only a single glomerulus. Thus, we propose that "one projection neuron-one glomerulus" is a basic principle used by various animal species including mouse, zebrafish, and Drosophila for odor information coding in the first relay station along the olfactory circuitry.

We observed that each MC projects its axon to multiple target regions in the forebrain, including bilateral hemispheres of the telencephalon and the right habenular nucleus. A similar divergent projection of single MCs is observed also in other species (Ojima et al., 1984; Marin et al., 2002; Wong et al., 2002) and is a striking contrast to the axon projection of each OSN to a single glomerulus. Divergent projections are also observed at a population level: MCs innervating the same glomerulus do not necessarily exhibit a similar projection pattern. Among the five singlelabeled mG1-MCs, for instance, four mG1-MCs showed the same target selectivity (bilateral telencephalic fields and right habenula) and similar branching complexity in the telencephalon, whereas the other exhibited no habenular projection and a more complicated branching pattern in the telencephalon. Hence, the odor information converged into glomeruli is delivered by MC axons to multiple brain areas in parallel and might be processed in different ways to execute distinct output responses. In addition to the divergent projections, we observed convergent projections of MCs axons. MCs innervating different glomeruli within the $\mathrm{mG}$ cluster frequently exhibit the axonal projection to a restricted portion in the right habenula as well as to the bilateral telencephalic fields. Thus, the odor information from different glomeruli within the $\mathrm{mG}$ cluster appears to be integrated onto a specific population of habenular neurons. Moreover, MCs innervating the $\mathrm{mG}$ cluster arborize axons in the telencephalic fields with considerable overlaps, providing third-order neurons in the telencephalon with a chance of integration of multiple inputs from different mGs.

Whether or not MCs project axons to the right habenula depends on which glomerular clusters the MC dendrites innervate. The majority of $\mathrm{mG}-\mathrm{MCs}$ project axons to the habenula, whereas the majority of MCs innervating outside the $\mathrm{mG}$ cluster do not, suggesting that the neurons in the right habenula may receive and process some particular odor information to elicit a specific behavioral response. In contrast, all the labeled MCs elaborate axonal branches in the telencephalon, regardless of glomerular clusters. A recent electrophysiological study in catfish showed that third-order neurons responsive for odorants relevant to feeding and social behaviors are topographically organized in different regions of the telencephalon (Nikonov et al., 2005). In this study, we found that MCs innervating different glomerular clusters ( $\mathrm{mG}, \mathrm{lG}$ and $\mathrm{avG}$ ) tend to show axonal terminations in distinct but partly overlapping telencephalic regions, presumably reflecting the topographic organization of odor representations in the telencephalon to some extent.

\section{Direct and asymmetric bulbo-habenular projection: functional insights}

The habenular nuclei are a bilaterally paired structure in the epithalamus, which constitute the dorsal diencephalic conduction pathway connecting the limbic forebrain and the midbrain. Anatomical left-right asymmetries of the habenulae have been described for a wide range of animal species from fish to mammals (Concha and Wilson, 2001). In zebrafish, the habenular asymmetry extends to differences in gene expression, subnuclear organization, neural connectivity, and timing of neurogenesis (Concha and Wilson, 2001; Aizawa et al., 2005; Gamse et al., 2005; Aizawa et al., 2007; Bianco et al., 2008). Zebrafish display a variety of lateralized behaviors with regard to the preferential eye usage (Miklósi and Andrew, 2006). For instance, zebrafish use the right eye when approaching and biting a target, whereas they use the left eye when assessing whether an object is identical with that seen before. The mutant zebrafish, frequent-situs-inversus $\left(f_{s i}\right)$, with reversed laterality of the habenulae show opposite preferences of eye usage in some behavioral tasks (Barth et al., 2005). Furthermore, many fish species including zebrafish show a consistent lateral bias at a population level in turning response during predator-escape behaviors upon visual stimuli (Bisazza et al., 2000). Thus, brain asymmetry underlies various lateralized behaviors of vertebrates.

Our data clearly demonstrate the asymmetric axonal projection from the OB to the habenula in zebrafish. The MCs innervating the $\mathrm{mG}$ cluster in bilateral OBs send axons only to the right habenula, converting the odor inputs from bilateral nostrils into the unilateral information within the brain through this asymmetric axonal wiring. Because the habenulae constitute the limbic-system output pathway for evoking emotional and social behaviors, it is tempting to speculate that predator-escape responses not only upon visual stimuli but also upon olfactory stimuli might show a lateralization, and if so, it might be mediated by the asymmetric bulbo-habenular circuitry. This prediction is also supported by the direct, monosynaptic projection from the $\mathrm{OB}$ to the right habenula that probably leads to an urgent behavioral response with no requirement of information processing in the telencephalon. A recent study demonstrated that a hard-wired neural circuit involving a discrete glomerular cluster in the dorsal OB take charge of the innate aversive/fear behaviors in mice (Kobayakawa et al., 2007). The convergent projection of $\mathrm{mG}-\mathrm{MCs}$ to the right habenula in zebrafish would be consistent with the nature of neural circuits dedicated for innate behaviors. Genetic ablation or inactivation of OSNs and/or MCs innervating the $\mathrm{mG}$ cluster will clarify the functional importance of lateralized bulbo-habenular projection.

\section{References}

Aizawa H, Bianco IH, Hamaoka T, Miyashita T, Uemura O, Concha ML, Russell C, Wilson SW, Okamoto H (2005) Laterotopic representation of left-right information onto the dorso-ventral axis of a zebrafish midbrain target nucleus. Curr Biol 15:238-243.

Aizawa H, Goto M, Sato T, Okamoto H (2007) Temporally regulated asymmetric neurogenesis causes left-right difference in the zebrafish habenular structures. Dev Cell 12:87-98.

Ando H, Kobayashi M, Tsubokawa T, Uyemura K, Furuta T, Okamoto H (2005) Lhx2 mediates the activity of Six3 in zebrafish forebrain growth. Dev Biol 287:456-468.

Baier H, Korsching S (1994) Olfactory glomeruli in the zebrafish form an invariant pattern and are identifiable across animals. J Neurosci 14:219-230.

Barth KA, Miklosi A, Watkins J, Bianco IH, Wilson SW, Andrew RJ (2005) fsi zebrafish show concordant reversal of laterality of viscera, neuroanatomy, and a subset of behavioral responses. Curr Biol 15:844-850.

Bianco IH, Carl M, Russell C, Clarke JD, Wilson SW (2008) Brain asymmetry is encoded at the level of axon terminal morphology. Neural Dev 3:9.

Bisazza A, Cantalupo C, Capocchiano M, Vallortigara G (2000) Population lateralisation and social behaviour: a study with 16 species of fish. Laterality 5:269-284. 
Castro A, Becerra M, Manso MJ, Anadón R (2006) Calretinin immunoreactivity in the brain of the zebrafish, Danio rerio: distribution and comparison with some neuropeptides and neurotransmitter-synthesizing enzymes. I. Olfactory organ and forebrain. J Comp Neurol 494:435-459.

Chess A, Simon I, Cedar H, Axel R (1994) Allelic inactivation regulates olfactory receptor gene expression. Cell 78:823-834.

Concha ML, Wilson SW (2001) Asymmetry in the epithalamus of vertebrates. J Anat 199:63-84.

Dryer L, Graziadei PP (1994) Mitral cell dendrites: a comparative approach. Anat Embryol (Berl) 189:91-106.

Dynes JL, Ngai J (1998) Pathfinding of olfactory neuron axons to stereotyped glomerular targets revealed by dynamic imaging in living zebrafish embryos. Neuron 20:1081-1091.

Friedrich RW, Korsching SI (1997) Combinatorial and chemotopic odorant coding in the zebrafish olfactory bulb visualized by optical imaging. Neuron 18:737-752.

Friedrich RW, Korsching SI (1998) Chemotopic, combinatorial, and noncombinatorial odorant representations in the olfactory bulb revealed using a voltage-sensitive axon tracer. J Neurosci 18:9977-9988.

Fuller CL, Byrd CA (2005) Ruffed cells identified in the adult zebrafish olfactory bulb. Neurosci Lett 379:190-194.

Fuller CL, Yettaw HK, Byrd CA (2006) Mitral cells in the olfactory bulb of adult zebrafish (Danio rerio): morphology and distribution. J Comp Neurol 499:218-230.

Gamse JT, Kuan YS, Macurak M, Brösamle C, Thisse B, Thisse C, Halpern ME (2005) Directional asymmetry of the zebrafish epithalamus guides dorsoventral innervation of the midbrain target. Development 132:4869-4881.

Hansen A, Zeiske E (1998) The peripheral olfactory organ of the zebrafish, Danio rerio: an ultrastructural study. Chem Senses 23:39-48.

Hashiguchi Y, Nishida M (2007) Evolution of trace amine associated receptor (TAAR) gene family in vertebrates: lineage-specific expansions and degradations of a second class of vertebrate chemosensory receptors expressed in the olfactory epithelium. Mol Biol Evol 24:2099-2107.

Higashijima S, Mandel G, Fetcho JR (2004) Distribution of prospective glutamatergic, glycinergic, and GABAergic neurons in embryonic and larval zebrafish. J Comp Neurol 480:1-18.

Jefferis GS, Potter CJ, Chan AM, Marin EC, Rohlfing T, Maurer CR Jr, Luo L (2007) Comprehensive maps of Drosophila higher olfactory centers: spatially segregated fruit and pheromone representation. Cell 128:1187-1203.

Kimura N, Nakashima K, Ueno M, Kiyama H, Taga T (1999) A novel mammalian T-box-containing gene, Tbr2, expressed in mouse developing brain. Brain Res Dev Brain Res 115:183-193.

Kobayakawa K, Kobayakawa R, Matsumoto H, Oka Y, Imai T, Ikawa M, Okabe M, Ikeda T, Itohara S, Kikusui T, Mori K, Sakano H (2007) Innate versus learned odour processing in the mouse olfactory bulb. Nature 450:503-508.

Kosaka T (1980) Ruffed cell: a new type of neuron with a distinctive initial unmyelinated portion of the axon in the olfactory bulb of the goldfish (Carassius auratus): II. Fine structure of the ruffed cell. J Comp Neurol 193:119-145.

Kosaka T, Hama K (1979) Ruffed cell: a new type of neuron with a distinctive initial unmyelinated portion of the axon in the olfactory bulb of the goldfish (Carassius auratus) I. Golgi impregnation and serial thin sectioning studies. J Comp Neurol 186:301-319.

Li J, Mack JA, Souren M, Yaksi E, Higashijima S, Mione M, Fetcho JR, Friedrich RW (2005) Early development of functional spatial maps in the zebrafish olfactory bulb. J Neurosci 25:5784-5795.

Lin HH, Lai JS, Chin AL, Chen YC, Chiang AS (2007) A map of olfactory representation in the Drosophila mushroom body. Cell 128:1205-1217.
Malnic B, Hirono J, Sato T, Buck LB (1999) Combinatorial receptor codes for odors. Cell 96:713-723.

Marin EC, Jefferis GS, Komiyama T, Zhu H, Luo L (2002) Representation of the glomerular olfactory map in the Drosophila brain. Cell 109:243-255.

Miklósi A, Andrew RJ (2006) The zebrafish as a model for behavioral studies. Zebrafish 3:227-234.

Mione M, Shanmugalingam S, Kimelman D, Griffin K (2001) Overlapping expression of zebrafish T-brain-1 and eomesodermin during forebrain development. Mech Dev 100:93-97.

Miyasaka N, Sato Y, Yeo SY, Hutson LD, Chien CB, Okamoto H, Yoshihara Y (2005) Robo2 is required for establishment of a precise glomerular map in the zebrafish olfactory system. Development 132:1283-1293.

Mombaerts P, Wang F, Dulac C, Chao SK, Nemes A, Mendelsohn M, Edmondson J, Axel R (1996) Visualizing an olfactory sensory map. Cell 87:675-686.

Mori K, Nagao H, Yoshihara Y (1999) The olfactory bulb: coding and processing of odor molecule information. Science 286:711-715.

Nikonov AA, Finger TE, Caprio J (2005) Beyond the olfactory bulb: an odotopic map in the forebrain. Proc Natl Acad Sci USA 102:18688-18693.

Ojima H, Mori K, Kishi K (1984) The trajectory of mitral cell axons in the rabbit olfactory cortex revealed by intracellular HRP injection. J Comp Neurol 230:77-87.

Ressler KJ, Sullivan SL, Buck LB (1994) Information coding in the olfactory system: evidence for a stereotyped and highly organized epitope map in the olfactory bulb. Cell 79:1245-1255.

Rubin BD, Katz LC (1999) Optical imaging of odorant representations in the mammalian olfactory bulb. Neuron 23:499-511.

Saraiva LR, Korsching SI (2007) A novel olfactory receptor gene family in teleost fish. Genome Res 17:1448-1457.

Sato Y, Miyasaka N, Yoshihara Y (2005) Mutually exclusive glomerular innervation by two distinct types of olfactory sensory neurons revealed in transgenic zebrafish. J Neurosci 25:4889-4897.

Sato Y, Miyasaka N, Yoshihara Y (2007) Hierarchical regulation of odorant receptor gene choice and subsequent axonal projection of olfactory sensory neurons in zebrafish. J Neurosci 27:1606-1615.

Serizawa S, Miyamichi K, Nakatani H, Suzuki M, Saito M, Yoshihara Y, Sakano H (2003) Negative feedback regulation ensures the one receptor-one olfactory neuron rule in mouse. Science 302:2088-2094.

Smear MC, Tao HW, Staub W, Orger MB, Gosse NJ, Liu Y, Takahashi K, Poo MM, Baier H (2007) Vesicular glutamate transport at a central synapse limits the acuity of visual perception in zebrafish. Neuron 53:65-77.

Speca DJ, Lin DM, Sorensen PW, Isacoff EY, Ngai J, Dittman AH (1999) Functional identification of a goldfish odorant receptor. Neuron 23:487-498.

Uchida N, Takahashi YK, Tanifuji M, Mori K (2000) Odor maps in the mammalian olfactory bulb: domain organization and odorant structural features. Nat Neurosci 3:1035-1043.

Vassar R, Chao SK, Sitcheran R, Nuñez JM, Vosshall LB, Axel R (1994) Topographic organization of sensory projections to the olfactory bulb. Cell 79:981-991.

Whitlock KE (2004) Development of the nervus terminalis: origin and migration. Microsc Res Tech 65:2-12.

Wong AM, Wang JW, Axel R (2002) Spatial representation of the glomerular map in the Drosophila protocerebrum. Cell 109:229-241.

Yoshihara Y, Kawasaki M, Tamada A, Fujita H, Hayashi H, Kagamiyama H, Mori K (1997) OCAM: A new member of the neural cell adhesion molecule family related to zone-to-zone projection of olfactory and vomeronasal axons. J Neurosci 17:5830-5842. 